Journal:

Article type: $\quad$ Original Article

\title{
Title: $\quad$ Heart failure following blood cancer therapy in pediatric and adult populations
}

\section{Short title: Heart failure after blood cancer therapy}

Julie Franzon ${ }^{1}$, Narelle M Berry ${ }^{1,2}$, Shahid Ullah ${ }^{3}$, Vincent L Versace ${ }^{4}$, John Atherton ${ }^{5}$, David Roder ${ }^{6}$, Bogda Koczwara ${ }^{7}$, Douglas Coghlan ${ }^{8}$, Robyn A Clark ${ }^{1}$

${ }^{1}$ School of Nursing \& Midwifery, Flinders University, Adelaide, South Australia, Australia

${ }^{2}$ Norwich Medical School, University of East Anglia, Norwich, United Kingdom

${ }^{3}$ South Australian Health and Medical Research Institute, Adelaide, South Australia, Australia

${ }^{4}$ Deakin Rural Health, School of Medicine, Deakin University, Warrnambool, Victoria, Australia

${ }^{5}$ Cardiology Department, Royal Brisbane and Women's Hospital and University of Queensland School of Medicine, Brisbane, Queensland, Australia

${ }^{6}$ Cancer Epidemiology and population Health Unit, School of Population Health, University of South Australia, Adelaide, South Australia, Australia

${ }^{7}$ Medical Oncology Unit, Flinders Centre for Innovation in Cancer, Flinders University and Flinders Medical Centre, Adelaide, South Australia, Australia

${ }^{8}$ Molecular Medicine and Pathology, Haematology, Flinders University, Adelaide, South Australia, Australia

\section{Corresponding Author:}

Dr Julie Franzon

School of Nursing and Midwifery, Flinders University

GPO Box 2100

Adelaide 5001

South Australia, Australia

Ph: $\quad+61882013066$

Fax: $\quad+61882013905$

Email: julie.franzon@flinders.edu.au

\section{Acknowledgements}

This work was supported by the Institute of Health and Biomedical Innovation of Queensland University of Technology under Collaborative Research Development Grant Scheme 2011 and a Flinders University Faculty of Health Sciences Seeding grant 2013.

Professor Robyn Clark is supported by a Heart Foundation Future Leader Fellowship (App ID:100847).

The authors acknowledge the contribution of Mr Munir Chowdhury to the earlier data preparation upon which this analysis is built 


\section{$\underline{\text { Abstract }}$}

Aim: The link between chemotherapy treatment and cardiotoxicity is well established, particularly for adults with blood cancers. However, it is less clear for children. This analysis aimed to compare the trajectory and mortality of children and adults who received chemotherapy for blood cancers and were subsequently hospitalised for heart failure.

Methods: Linked data from the Queensland Cancer Registry, Death Registry and Hospital Administration records for initial chemotherapy and later heart failure were reviewed (19962009). Of all identified blood cancer patients $(N=23,434) ; 8,339$ received chemotherapy, including 817 children (aged $\leq 18$ years at time of cancer diagnosis) and 7,522 adults. Timevarying Cox proportional hazards regression models were used to compare the characteristics and survival between the two groups.

Results: Of those who were subsequently hospitalised for heart failure, $70 \%$ of children and $46 \%$ of adults had the index admission within 12 months of their cancer diagnosis. Of these, $53 \%$ of the pediatric heart failure population and $71 \%$ of the adult heart failure population died within the study period. Following adjustment for age, sex and chemotherapy admissions, children with heart failure had an increased mortality risk compared to their nonheart failure counterparts, a difference which was much greater than that between the adult groups.

Conclusion: The impact of heart failure on children previously treated for blood cancer is more severe than for adults, with earlier morbidity and greater mortality. Improved strategies are needed for the prevention and management of cardiotoxicity in this population.

Keywords: cardiology, cardiotoxicity, chemotherapy, heart failure, adult, pediatric 


\section{Introduction}

Cardiotoxicity is a well-established adverse consequence of cancer treatment, with drugs used to treat blood cancers known to have long-term cardiovascular effects including left ventricular dysfunction, myocardial ischemia, hypertension and QTc prolongation. ${ }^{1,2}$ These cardiotoxic agents are widely used in both children ${ }^{3}$ and adults. ${ }^{4}$ In the general population, the median age of heart failure (HF) diagnosis typically ranges from 70 to 83 years and is extremely uncommon in children. ${ }^{5-8}$ However, children previously treated for cancer are 5.9 times more likely to develop congestive heart failure than their nearest age sibling. ${ }^{9}$ Furthermore, in the absence of overt HF in childhood, even small changes in ejection fraction from chemotherapy in childhood predispose adult survivors of childhood cancer to HF as they age. ${ }^{10}$

Studies indicate that the pathogenesis underlying chemotherapy-related cardiovascular changes operates on a cellular level. ${ }^{11-13}$ While this affects people of all ages, it is likely that the impact of chemotherapy treatment on a heart which is still developing will be different to that on a mature organ. Research has shown that cardiotoxicity can manifest up to 20 years after cancer therapy, and with improving survival rates, particularly from childhood cancer, ${ }^{14,15}$ this impact is likely to become even more pronounced. ${ }^{16-18}$ This confers a need to further understand the characteristics of heart failure development in children previously treated for cancer. 
Previous work from our research group has demonstrated that in a cohort of patients with breast or blood cancer $(n=15,987), 6.6 \%$ of patients treated with chemotherapy were later hospitalised for heart failure. ${ }^{19}$ The median age of this population was 55.0 years (IQR, 44-65), and included 817 (5.1\%) patients aged 18 years or under. The purpose of this secondary analysis is to compare the characteristics and survival outcomes of children and adults in order to identify differences in the incidence, time to diagnosis and associated mortality rates. 


\section{Methods}

\section{Study design}

The parent study was a retrospective audit of linked health administration data from Queensland, Australia. Approval was granted by the Metro South Health Service District Human Research Ethics Committee (HREC/11/QPAH/600).

\section{Data sources and data linkage}

Three independent databases were linked to create the analysis database for this study: (1) the Queensland Cancer Registry (QCR), (2) the Queensland Hospital Admitted Patient Data Collection, and (3) the Queensland Birth, Deaths and Marriages Registry.

The QCR houses data from all persons admitted to public and private hospitals and nursing homes with a cancer diagnosis in Queensland since $1982 .{ }^{20}$ The initial population of interest was identified from this by selecting patients registered between January 1996 and December 2009 with a primary diagnosis of blood cancer. These dates were chosen to ensure a uniform time frame across all data sets.

The Hospital Admitted Patient Data Collection contains data on all hospital separations from any Queensland hospital permitted to admit patients. The QCR facility and Unit Record (UR) numbers were match-merged with admissions episodes from the same time period to identify those who received chemotherapy treatment. These records formed the study cohort. 
The hospital admissions were further interrogated to stratify the members of the cohort with an index HF separation versus those without. The index HF episode must have occurred after the date of cancer registry entry to be included in the HF group.

Finally, the study cohort records were linked to the Queensland Birth, Deaths and Marriages database - a repository for all registered deaths in Queensland. All-cause mortality data were then extracted to complete the database. LinkageWiz software was used for probabilistic matching. ${ }^{21}$

\section{Participants}

The primary cancer diagnosis recorded in the QCR was used to identify blood cancers using the International Classification of Diseases and Tenth Revision Australian Modification (ICD 10-AM) and ICD-O (oncology) site codes ICD10-AM: C42, C77 and ICD-O: M9590/3-M9989/3.

Patients prescribed chemotherapy were identified from the relevant codes in the hospital records, defined according to ICD-9-CM (clinical modification) (99.25) and ICD-10AM (Australian Modification) (96196-00, 96199-00, 96209-00, 96207-00, 96208-00, 96204-00, 13942-00, 13915-00, 13918-00, 13921-00, 13927-00, 13939-00, 13942-00, Z51.1, Z51.2).

Blood cancer patients who were identified as having received chemotherapy treatment were stratified by age group (children: aged 18 years and under; adult: over 18 years). 
Participants were then categorised into those who had an index HF admission after cancer diagnosis (HF group) compared with those who did not (non-HF group). An index HF admission was defined as a patient's first hospitalisation coded for HF. HF diagnosis was based on the ICD-9-CM (428.0. 428.1 and 428.9) and ICD-10-AM (I50.0, I50.1 and I50.9) codes and included Congestive HF, Left Ventricular failure and other unspecified cardiomyopathies.

\section{Outcome, exposure and confounders}

The primary outcomes were (1) mortality and (2) HF which occurred after blood cancer diagnosis and treatment; indicated by an index hospital admission coded for HF. Both incidence and the time to index HF admission and/or death were measured.

Possible confounders include demographic characteristics and clinical features such as income, education, medical history, chemotherapy agent and dose, and other treatment modalities such as transplantation or concurrent radiotherapy treatment.

\section{Statistical analysis}

Data management and statistical analyses were performed using STATA version 14.0 (StataCorp LP, College Station, TX, USA). Means and standard deviations (SD) for discrete and continuous data were calculated and categorical variables were presented as percentages. 
Differences in demographics and cancer treatment history were analysed for betweengroup differences using Fisher's exact and chi-square tests. Where continuous data were non-normally distributed, the data are presented as median (inter-quartile range) (IQR) and compared using appropriate non-parametric tests. Differences were considered significant at $\mathrm{p}<0.05$.

The time-varying Cox proportional hazards regression models were used to evaluate factors associated with mortality risk during follow-up within groups adjusted by demographic variables and cancer treatment history (Table 2). Hazard ratios (HR) were used to describe the probability of survival after chemotherapy treatment in each group. The proportional hazard assumptions as well as goodness of fit of the models were also tested.

Kaplan-Meier survival curves were derived to compare the time to death from QCR registration between HF and non-HF groups for both adults and children, adjusted for sex and age using a log-rank test. 


\section{Results}

\section{Participants}

The inclusion of participants is described in accordance with the STrengthening the Reporting of OBservational studies in Epidemiology (STROBE) flow chart ${ }^{22}$ (Figure S1).

A total of 30,612 hematologic cancer records in the QCR were linked to hospital admissions and death records. Within the QCR, there were 625 patients registered with multiple cancers - the primary cancer diagnosis was used and all other registrations were excluded.

6,553 records were excluded as they fell outside the study timeframe, and a further 15,095 records were excluded as these patients did not receive chemotherapy. The final sample consisted of $N=8,339$ patients; $N=817$ children, and $N=7,522$ adults. Of these, $N=30(3.7 \%)$ children and $N=852(11.3 \%)$ adults had an index $\mathrm{HF}$ admission recorded after their cancer diagnosis.

Of those in the child HF group, $N=16(53.3 \%)$ patients died within the 13 year study period, while in the non-HF group $N=114(14.5 \%)$ patients died within the study period. In the adult HF group, $N=601(70.5 \%)$ patients died within the study period compared to $N=2,414(36.2 \%)$ patients in the adult non-HF group. 


\section{Demographics and clinical characteristics}

Demographic information was extracted from the QCR (Table 1). Within the adult study population, there were significant differences in the median age at cancer diagnosis, the median number of chemotherapy admissions and the quintiles of chemotherapy admissions between those who developed HF and those who did not. However these differences were not observed in the child population (Table 1).

\section{Outcome data}

Of those who had an index HF admission after chemotherapy (children $N=30$ and adults $N=852$ ), $70.0 \%$ of the children compared to $46.4 \%$ of adult patients were admitted for HF within 12 months of their initial cancer diagnosis (Figure 1). Furthermore, a total of $83.3 \%$ of the children compared to $69.3 \%$ of adult patients were admitted for HF within the first 3 years of cancer diagnosis.

All-cause mortality (adjusted for age, sex, and chemotherapy admissions) differed between groups, with both the child HF group (HR 4.86 (95\% CI, 2.85-8.27), $P<0.001$ ) (Figure 2) and the adult HF group (HR 1.66 (95\% CI, 1.51-1.82), $P<0.001)$ (Figure 3) having a greater risk of mortality relative to the respective non-HF groups. Overall, the mortality rate was almost 4 times higher for children than for adults. 
The adjusted time-varying Cox Proportional Hazard modelling showed that both child and adult non-HF patients along with adult HF patients had an increased risk of mortality with increasing age (Table 2). This was not significant in the child HF patients, but this likely reflects the low numbers in this group and the HR (1.02) was higher than the HR (1.01) for the adult HF group. Although there were no differences in mortality risk by sex or number of chemotherapy admissions between the HF and nonHF child groups, there was a gender difference evident in the adult groups, with males having a higher risk of mortality for both the HF and non-HF groups than females (Table 2). 


\section{Discussion}

This secondary analysis aimed to compare the characteristics and clinical outcomes of both children aged $\leq 18$ years and adults who received chemotherapy treatment for blood cancer, and were later admitted for HF, relative to those who were not admitted for HF. The ongoing risk of HF and its associated mortality following chemotherapy have been previously described $^{2,4,13,3}$, although many prior studies were based on smaller datasets or did not incorporate comprehensive longitudinal follow up. We utilised data linkage to report the adjusted, long-term survival of an unselected cohort of patients who received chemotherapy for blood cancer in a state-wide database stratified according to whether or not they were later diagnosed with $\mathrm{HF}$.

The results of this study demonstrated that $3.7 \%$ of children and $11.3 \%$ of adults with chemotherapy-treated blood cancer had a later index admission for HF. These results are broadly consistent with other studies which have reported rates of cardiotoxicity following anthracycline treatment ranging from $0.9 \%-26 \%{ }^{23}$. Our results are likely to be underestimates, as we are reliant on hospital admission as an indicator for HF diagnosis and therefore cannot account for either sub-clinical presentation or HF treated in primary care only. The maximum 13 year follow up time of this study is insufficient to fully capture the late onset incidence of HF or HF-related death; especially in those who were treated for cancer as children; which is believed to occur up to 20 years after initial cancer treatment. ${ }^{3}$

While the adult population showed significant differences in both median age and number of chemotherapy separations between HF and non-HF groups, this analysis did not reach significance within the children. This is likely due to both the smaller sample size and the narrower age range of the children. 
Although the proportion of the children who were admitted for HF during the study period was lower than the adult sample $(3.7 \% \mathrm{v} 11.3 \%$ respectively, Table 1$)$, a high proportion of these recorded an index admission in the first 12 months after cancer diagnosis (Figure 1). Of the $30 \mathrm{HF}$ children, $70.0 \%(\mathrm{n}=21)$ had an index admission within the first 12 months of their cancer diagnosis compared to $46.4 \%(n=395)$ of the adults. For those children who were admitted for HF, there was an almost 4 times greater mortality risk than for children with cancer alone, significantly more than the difference between the adult groups where the risk was almost double in the HF group compared to the non-HF group. This may reflect the decreased likelihood that children have additional comorbidities or competing causes of death, such that the relative effect of developing HF will be more pronounced. Alternatively, it may be that their hearts are more sensitive to the effects of chemotherapy, or they are less likely to tolerate or respond to HF management.

While the current cardiac screening recommendations for individuals previously diagnosed with cancer and treated with known cardiotoxins include increased frequency of screening based on younger age at treatment and higher dosage of chemotherapy; ${ }^{11,24}$ even these precautions do not appear to be sufficient to mediate the differences in outcomes between children and adults who subsequently develop HF.

\section{Clinical Implications}

An observation from this study is the potentially high rate of early onset of HF detected in the pediatric HF population. While the absolute numbers are small (21 out of 30 children who had a HF admission had the index admission in the first 12 months after cancer diagnosis), the absolute incident rate of child HF admissions is half that seen in the adult HF population. However comparison with an age-matched general population cohort' (calculated using unpublished admissions data from SA Health, 2010-2012), reveals that while the adult HF admission incidence in the first 12 months after cancer treatment is over 200 times the 
population rate of HF admissions (adult HF 5,251 per 100,000 vs population 25.3 per $100,000)$, the pediatric HF admission incidence is over 600 times the population rate (pediatric HF 2,570 per 100,000 vs population 4.1 per 100,000). While the higher proportion of early onset cases in the children may reflect delayed diagnosis in the adult cohort, these incident rates suggest that there may be a significant pattern of early onset $\mathrm{HF}$ after cancer diagnosis in children vs adults. This pattern is not explicitly reported in the trials of management of hematologic malignancies where the focus is often on the long-term cardiac risk to patients. ${ }^{25-27}$ Furthermore, children are often excluded from clinical trials due to ethical concerns.

Current guidelines for the treatment of cancer in children recommend that cumulative anthracycline dose be limited to $\leq 250-300 \mathrm{mg} / \mathrm{m}^{2}$, with similar guidelines also given for other chemotherapy and radiation treatments. ${ }^{2,28}$ Survivorship guidelines also recommend cardiac monitoring for a number of years for both children and adults who have received cardiotoxic chemotherapy and radiotherapy. In children, recommended cardiac imaging frequency is anywhere from 1 to 5 yearly, depending on the age of the child at treatment, the cumulative chemotherapy dose, and whether radiotherapy treatment was also undertaken. ${ }^{28}$

While the balance between providing therapeutic levels of treatment and the risk of cardiotoxicity is difficult to navigate, this analysis reinforces the need for clinicians to consider both short term and long-term risk when deciding on chemotherapy dosage. While adverse effects might be unavoidable in some situations, the need for early and intensive monitoring - including during treatment - especially in children, is clearly necessary to reduce the severity and impact of subsequent cardiac events. Additional evidence around the optimal mode and frequency of cardiac monitoring is required to clarify best practice for each age group. Meanwhile rigorous research into the use of cardioprotectants, appropriate 
treatments for those with cardiac compromise, and the alternatives to cardiotoxic therapy, is also needed to build the evidence base for these therapies.

\section{Strengths and Limitations}

The strength of this study is the large sample size and the linkage process which enabled the identification of outcomes not usually recorded in isolated clinical data sets. While the results should be generalizable to any population of people who have been treated for blood cancers, there are some limitations to be considered beyond those already discussed above.

We were not able to ascertain precisely which chemotherapy treatments patients received or who also received radiotherapy. It is well known that different chemotherapy agents can have different cardiac effects, ${ }^{13,23}$ and that concurrent chest irradiation can also contribute to the development of cardiotoxicity, ${ }^{29,30}$ but these data were not available for linkage and could not be included in this analysis. Likewise we were not able to detect or control for any preexisting conditions which may predispose individuals to the development of heart failure. In the pediatric population, there was a short median follow-up time which was significantly different between the HF group and the non-HF group, with the non-HF group being followed for almost twice as long. However, this was largely explained by the shorter survival of the HF group with $50 \%$ dying within the study period compared to $14.5 \%$ of the non-HF group. Due to the short follow-up time for many patients, and existing evidence to suggest that adverse consequences of chemotherapy can present many years after treatment, the results of this study are likely to be an underestimate of the proportion of children who developed HF.

\section{Conclusion}

We identified different patterns of HF risk and trajectory between child and adult patients with blood cancer who received chemotherapy in this linked data set. The results of this study 
reinforce the need for regular cardiovascular screening of all patients, especially in the first three years following cancer diagnosis and treatment. Given the potential for the rapid onset of HF in children, even more frequent screening and the implementation of early detection protocols during treatment and throughout the first year may be necessary to allow for prompt action to reduce the impact of $\mathrm{HF}$, especially given the relatively poor outcomes for childhood cancer patients who develop HF. Future studies will also need to evaluate the uptake and tolerability of HF treatments, which have been largely informed from studies conducted in adult HF cohorts without cancer. 


\section{Authorship contributions}

Julie Franzon contributed to data analysis and interpretation, drafting the article, critical revision and final approval of the version to be published

Narelle M Berry contributed to the conception and design of the work, drafting the article, critical revision and final approval of the version to be published

Shahid Ullah contributed to data analysis and interpretation, critical revision and final approval of the version to be published

Vincent Versace contributed to data analysis and interpretation, drafting the article, critical revision and final approval of the version to be published

Alexandra L McCarthy contributed to the conception and design of the work, data collection, critical revision of the article and final approval of the version to be published

John Atherton contributed to the conception and design of the work, data collection, critical revision of the article and final approval of the version to be published

David Roder contributed to the conception and design of the work, data collection, critical revision of the article and final approval of the version to be published

Bogda Koczwara contributed to the conception and design of the work, data collection, critical revision of the article and final approval of the version to be published

Douglas Coghlan contributed to the conception and design of the work, critical revision of the article and final approval of the version to be published

Robyn A Clark contributed to the conception and design of the work, data collection, critical revision of the article and final approval of the version to be published

\section{Disclosure of conflicts of interest}

The authors have declared no conflicts of interest 


\section{References}

[1] Curigliano G, Cardinale D, Suter T, et al. Cardiovascular toxicity induced by chemotherapy, targeted agents and radiotherapy: ESMO Clinical Practice Guidelines. Ann Oncol 2012; 23: vii155vii66.

[2] Albini A, Pennesi G, Donatelli F, Cammarota R, De Flora S, Noonan DM. Cardiotoxicity of anticancer drugs: the need for cardio-oncology and cardio-oncological Prevention. J Natl Cancer Inst 2010; 102: 14-25.

[3] Kremer LC, Caron HN. Anthracycline cardiotoxicity in children. N Engl J Med 2004; 351: $120-21$.

[4] Cardinale D, Colombo A, Bacchiani G, et al. Early Detection of Anthracycline Cardiotoxicity and Improvement With Heart Failure Therapy. Circulation 2015; 131: 1981.

[5] Bleumink GS, Knetsch AM, Sturkenboom MC, et al. Quantifying the heart failure epidemic: prevalence, incidence rate, lifetime risk and prognosis of heart failure The Rotterdam Study. Eur Heart J 2004; 25: 1614-19.

[6] Cowie MR, Wood DA, Coats AJS, et al. Incidence and aetiology of heart failure; a population-based study. Eur Heart J 1999; 20: 421-28.

[7] Ho KKL, Pinsky JL, Kannel WB, Levy D, Pitt B. The epidemiology of heart failure: The Framingham Study. J Am Coll Cardiol 1993; 22: A6-A13.

[8] Teng TH, Finn J, Hobbs M, Hung J. Heart failure: incidence, case fatality, and hospitalization rates in Western Australia between 1990 and 2005. Circ Heart Fail 2010; 3: 236-43.

[9] Mulrooney DA, Yeazel MW, Kawashima T, et al. Cardiac outcomes in a cohort of adult survivors of childhood and adolescent cancer: retrospective analysis of the Childhood Cancer Survivor Study cohort. BMJ 2009; 339: b4606.

[10] Lipshultz SE, Franco VI, Cochran TR. Cardiotoxicity in childhood cancer survivors: a problem with long-term consequences in need of early detection and prevention. Pediatr Blood Cancer 2013; 60: 1395-6. 
[11] Giantris A, Abdurrahman L, Hinkle A, Asselin B, Lipshultz SE. Anthracycline-induced cardiotoxicity in children and young adults. Crit Rev Oncol Hematol 1998; 27: 53-68.

[12] Harake D, Franco VI, Henkel JM, Miller TL, Lipshultz SE. Cardiotoxicity in childhood cancer survivors: strategies for prevention and management. Future Cardiol 2012; 8: 647-70.

[13] Lipshultz SE, Adams MJ, Colan SD, et al. Long-term Cardiovascular Toxicity in Children, Adolescents, and Young Adults Who Receive Cancer Therapy: Pathophysiology, Course, Monitoring, Management, Prevention, and Research Directions. Circulation 2013; 128: 1927.

[14] Australian Institute of Health and Welfare. Cancer in Australia: an overview 2014.). Cancer series No 90 Cat no CAN 88. Canberra: AIHW, 2014.

[15] Ward E, DeSantis C, Robbins A, Kohler B, Jemal A. Childhood and adolescent cancer statistics, 2014. CA Cancer J Clin 2014; 64: 83-103.

[16] Cardinale D, Colombo A, Lamantia G, et al. Anthracycline-induced cardiomyopathy: clinical relevance and response to pharmacologic therapy. J Am Coll Cardiol 2010; 55: 213-20.

[17] Fulbright JM. Review of Cardiotoxicity in Pediatric Cancer Patients: During and after Therapy. Cardiol Res Pract 2011; 2011: 9.

[18] Wells QS, Lenihan DJ. Reversibility of left ventricular dysfunction resulting from chemotherapy: can this be expected? Prog Cardiovasc Dis 2010; 53: 140-48.

[19] Clark RA, Berry NM, Chowdhury MH, et al. Heart failure following cancer treatment: characteristics, survival and mortality of a linked health data analysis. Intern Med J 2016; 46: 1297 306.

[20] AIHW. Queensland Cancer Registry.[Australian Institute of Health and Welfare, Canberra 2013[updated cited 06/01/2015.Available from: http://www.aihw.gov.au/qld-cancer-registry/.

[21] State of Queensland. Queensland Data Linkage Framework. In: Health) SoQQ (ed)).

Queensland, Australia: State of Queensland (Queensland Health), 2014.

[22] Vandenbroucke JP, Von Elm E, Altman DG, et al. Strengthening the Reporting of Observational Studies in Epidemiology (STROBE): explanation and elaboration. Ann Intern Med 2007; 147: W-163-W-94. 
[23] Yeh ET, Bickford CL. Cardiovascular complications of cancer therapy: incidence, pathogenesis, diagnosis, and management. J Am Coll Cardiol 2009; 53: 2231-47.

[24] Armenian SH, Hudson MM, Mulder RL, et al. Recommendations for cardiomyopathy surveillance for survivors of childhood cancer: a report from the International Late Effects of Childhood Cancer Guideline Harmonization Group. The Lancet Oncology 2015; 16: e123-e36. [25] Pellicori P, Calicchia A, Lococo F, Cimino G, Torromeo C. Subclinical anthracycline cardiotoxicity in patients with acute promyelocytic leukemia in long-term remission after the AIDA protocol. Congest Heart Fail 2012; 18: 217-21.

[26] Vandecruys E, Mondelaers V, De Wolf D, Benoit Y, Suys B. Late cardiotoxicity after low dose of anthracycline therapy for acute lymphoblastic leukemia in childhood. J Cancer Surviv 2012; 6: $95-101$.

[27] Sorensen K, Levitt GA, Bull C, Dorup I, Sullivan ID. Late anthracycline cardiotoxicity after childhood cancer: a prospective longitudinal study. Cancer 2003; 97: 1991-8.

[28] Children's Oncology Group. Long-Term Follow-Up Guidelines for Survivors of Childhood, Adolescent and Young Adult Cancers, Version 4.0.[Children's Oncology Group, Monrovia, CA 2013[updated cited December 2016.Available from: http://www.survivorshipguidelines.org/pdf/LTFUGuidelines_40.pdf.

[29] Berry GJ, Jorden M. Pathology of radiation and anthracycline cardiotoxicity. Pediatr Blood Cancer 2005; 44: 630-7.

[30] Bovelli D, Plataniotis G, Roila F, Group EGW. Cardiotoxicity of chemotherapeutic agents and radiotherapy-related heart disease: ESMO Clinical Practice Guidelines. Ann Oncol 2010; 21 Suppl 5: v277-v82. 
Table 1 Demographics and chemotherapy separation rates for adults ( $>18$ years $)$ and children ( $\leq 18$ years) who received chemotherapy for blood cancer

\begin{tabular}{|c|c|c|c|c|c|c|}
\hline \multirow[b]{2}{*}{ Characteristics } & \multicolumn{3}{|c|}{ Adults $N=7,522(90.2 \%)$} & \multicolumn{3}{|c|}{ Children $N=817(9.8 \%)$} \\
\hline & $\begin{array}{c}\text { Heart Failure } \\
N=\mathbf{8 5 2}(11.3 \%)\end{array}$ & $\begin{array}{c}\text { Non-HF } \\
N=\mathbf{6 , 6 7 0}(\mathbf{8 8 . 7 \%})\end{array}$ & P-value & $\begin{array}{l}\text { Heart Failure } \\
N=30(3.7 \%)\end{array}$ & $\begin{array}{c}\text { Non-HF } \\
N=787(96.3 \%)\end{array}$ & P-value \\
\hline $\begin{array}{l}\text { Age (at cancer diagnosis), years } \\
\text { Median (IQR) }\end{array}$ & $68(60-75)$ & $60(48-69)$ & $P<0.001$ & $5(3-12)$ & $7(3-14)$ & $P=0.51$ \\
\hline $\operatorname{Sex}, N(\%)$ & & & $P=0.72$ & & & $P=0.62$ \\
\hline Female & $357(41.9)$ & $2,752(41.3)$ & & $12(40.0)$ & $351(44.6)$ & \\
\hline Male & $495(58.1)$ & $3,918(58.7)$ & & $18(60.0)$ & $436(55.4)$ & \\
\hline Marital Status, n(\%) & & & $P=0.01$ & & & - \\
\hline Married/De Facto & $532(62.4)$ & $4,459(66.8)$ & & - & $13(1.2)$ & \\
\hline Single/divorced/widowed & 320 (37.6) & $2,211(33.2)$ & & $30(100.0)$ & $774(98.4)$ & \\
\hline Country of Birth, n(\%) & & & $P<0.01$ & & & \\
\hline Australia & $602(70.7)$ & $4,992(74.9)$ & & $30(100.0)$ & $723(91.9)$ & - \\
\hline All Other Countries & $250(29.3)$ & $1,678(25.2)$ & & - & $64(8.1)$ & \\
\hline Residence (Postcode), $N(\%)$ & & & $P=0.60$ & & & $P=0.94$ \\
\hline Metropolitan & $734(86.2)$ & $5,789(86.8)$ & & $26(86.7)$ & $678(86.1)$ & \\
\hline Rural/Remote & $118(13.9)$ & $881(13.2)$ & & $4(13.3)$ & $109(13.9)$ & \\
\hline $\begin{array}{l}\text { No of chemotherapy separations, } \\
\text { Median (IQR) }\end{array}$ & $5(2-11)$ & $7(3-14)$ & $P<0.001$ & $16(4-35)$ & $19(8-36)$ & $P=0.36$ \\
\hline $\begin{array}{l}\text { No of chemotherapy separations, } \\
\text { Quintiles }\end{array}$ & & & $P<0.001$ & & & $P=0.08$ \\
\hline $1-3$ & $357(41.9)$ & $1,941(29.1)$ & & $7(23.3)$ & $80(10.2)$ & \\
\hline $4-6$ & $165(19.4)$ & $1,309(19.6)$ & & $4(13.3)$ & $71(9.0)$ & \\
\hline $7-9$ & $97(11.4)$ & $901(13.5)$ & & - & $76(9.6)$ & \\
\hline $10-16$ & $132(15.5)$ & $1,351(20.3)$ & & $5(16.7)$ & $154(19.6)$ & \\
\hline$\geq 17$ & $101(11.9)$ & $1,168(17.5)$ & & $14(46.7)$ & $406(51.6)$ & \\
\hline
\end{tabular}

IQR = interquartile range (25th-75th percentile)

Significant $\leq 0.05$ 
Table 2 Adjusted time-varying Cox Proportional Hazard Models for all-cause mortality between HF and non-HF groups for adults and children. Adjusted for age, sex, marital status, country of birth and number of chemotherapy admissions

\begin{tabular}{|c|c|c|c|c|c|c|c|c|}
\hline \multirow[b]{2}{*}{ Parameter } & \multicolumn{4}{|c|}{ Adults $N=7,522(90.2 \%)$} & \multicolumn{4}{|c|}{ Children $N=817(9.8 \%)^{\mathrm{a}}$} \\
\hline & $\begin{array}{l}\text { Heart Failure } \\
\text { HR }(95 \% \text { CI })\end{array}$ & $P$-value & $\begin{array}{c}\text { Non-HF } \\
\text { HR }(95 \% \text { CI })\end{array}$ & $P$-value & $\begin{array}{l}\text { Heart Failure } \\
\text { HR }(95 \% \text { CI })\end{array}$ & $P$-value & $\begin{array}{c}\text { Non-HF } \\
\text { HR }(95 \% \text { CI })\end{array}$ & $P$-value \\
\hline $\begin{array}{l}\text { Age (at cancer diagnosis), } \\
\text { Years }\end{array}$ & $1.01(1.01-1.02)$ & $P<0.01$ & $1.03(1.03-1.04)$ & $P<0.001$ & $1.02(0.91-1.15)$ & $P=0.73$ & $1.04(1.01-1.08)$ & $P=0.01$ \\
\hline $\begin{array}{l}\text { Sex } \\
\text { Female vs Male }\end{array}$ & $1.30(1.10-1.54)$ & $P<0.01$ & $1.28(1.17-1.39)$ & $P<0.001$ & $1.09(0.39-3.09)$ & $P=0.87$ & $0.88(0.61-1.27)$ & $P=0.50$ \\
\hline $\begin{array}{l}\text { Marital Status } \\
\text { Married/De Facto vs All other }\end{array}$ & $1.16(0.97-1.38)$ & $P=0.10$ & $1.22(1.12-1.32)$ & $P<0.001$ & - & - & - & - \\
\hline $\begin{array}{l}\text { Country of Birth } \\
\text { All Other vs Australia }\end{array}$ & $0.77(0.64-0.92)$ & $P<0.01$ & $1.06(0.97-1.16)$ & $P=0.23$ & - & - & - & - \\
\hline $\begin{array}{l}\text { No of chemotherapy admission } \\
1-3 \\
4-6 \\
7-9 \\
10-16 \\
\geq 17 \\
\end{array}$ & $\begin{array}{c}\text { Referent } \\
1.00(0.95-1.07) \\
0.99(0.92-1.06) \\
0.96(0.90-1.02) \\
0.98(0.92-1.05) \\
\end{array}$ & $\begin{array}{c}- \\
P=0.89 \\
P=0.70 \\
P=0.21 \\
P=0.54\end{array}$ & $\begin{array}{c}\text { Referent } \\
0.91(0.88-0.04) \\
0.93(0.89-0.96) \\
0.94(0.91-0.97) \\
0.99(0.96-1.02) \\
\end{array}$ & $\begin{array}{c}- \\
P<0.001 \\
P<0.001 \\
P<0.001 \\
P=0.37\end{array}$ & $\begin{array}{c}\text { Referent } \\
0.83(0.25-2.81) \\
- \\
1.72(0.50-5.85) \\
1.05(0.38-2.95) \\
\end{array}$ & $\begin{array}{c}P=0.77 \\
- \\
P=0.39 \\
P=0.92\end{array}$ & $\begin{array}{c}\text { Referent } \\
0.66(0.39-1.11) \\
0.86(0.63-1.17) \\
0.87(0.66-1.13) \\
0.91(0.72-1.15) \\
\end{array}$ & $\begin{array}{l}P=0.11 \\
P=0.33 \\
P=0.29 \\
P=0.45\end{array}$ \\
\hline
\end{tabular}

$\mathrm{HR}=$ hazard ratio

$\mathrm{CI}=$ confidence interval 


\section{Figure legends}

Figure 1 Time from cancer diagnosis to the index heart failure admission in $N=30$ children and $\mathrm{N}=852$ adults who underwent chemotherapy treatment for blood cancer and were later admitted for heart failure

Figure 2 Survival curves for all-cause mortality of HF and non-HF chemotherapy-receiving childhood cancer patients, adjusted by age, sex and number of chemotherapy separations

Figure 3 Survival curves for all-cause mortality of HF and non-HF chemotherapy-receiving adult cancer patients, adjusted by age, sex, marital status, country of birth and number of chemotherapy separations 
Figure 1 Time from cancer diagnosis to the index heart failure admission in $N=30$ children and $N=852$ adults who underwent chemotherapy treatment for blood cancer and were later admitted for heart failure

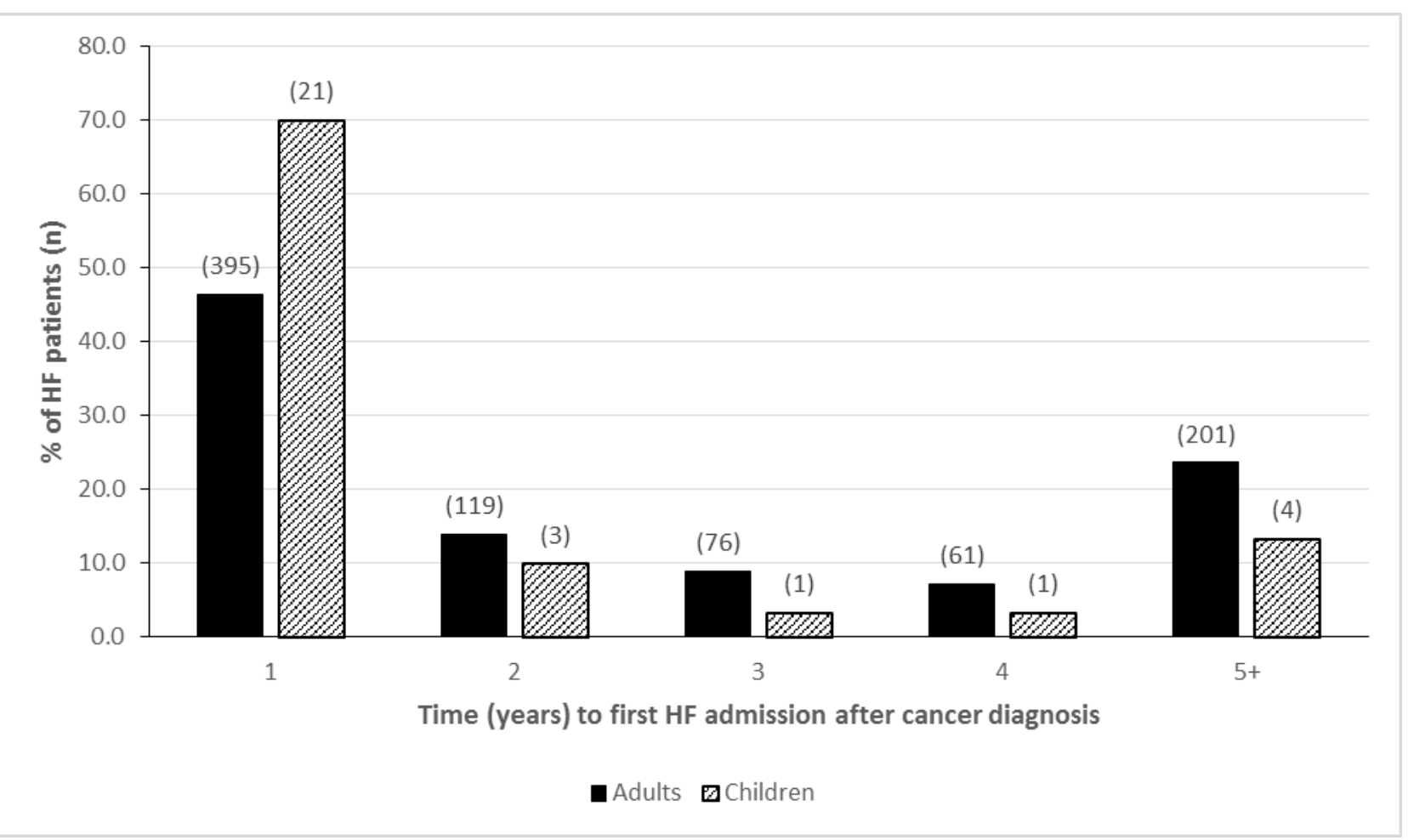


Figure 2 Survival curves for all-cause mortality of HF and non-HF chemotherapy-receiving childhood cancer patients, adjusted by age, sex and number of chemotherapy separations

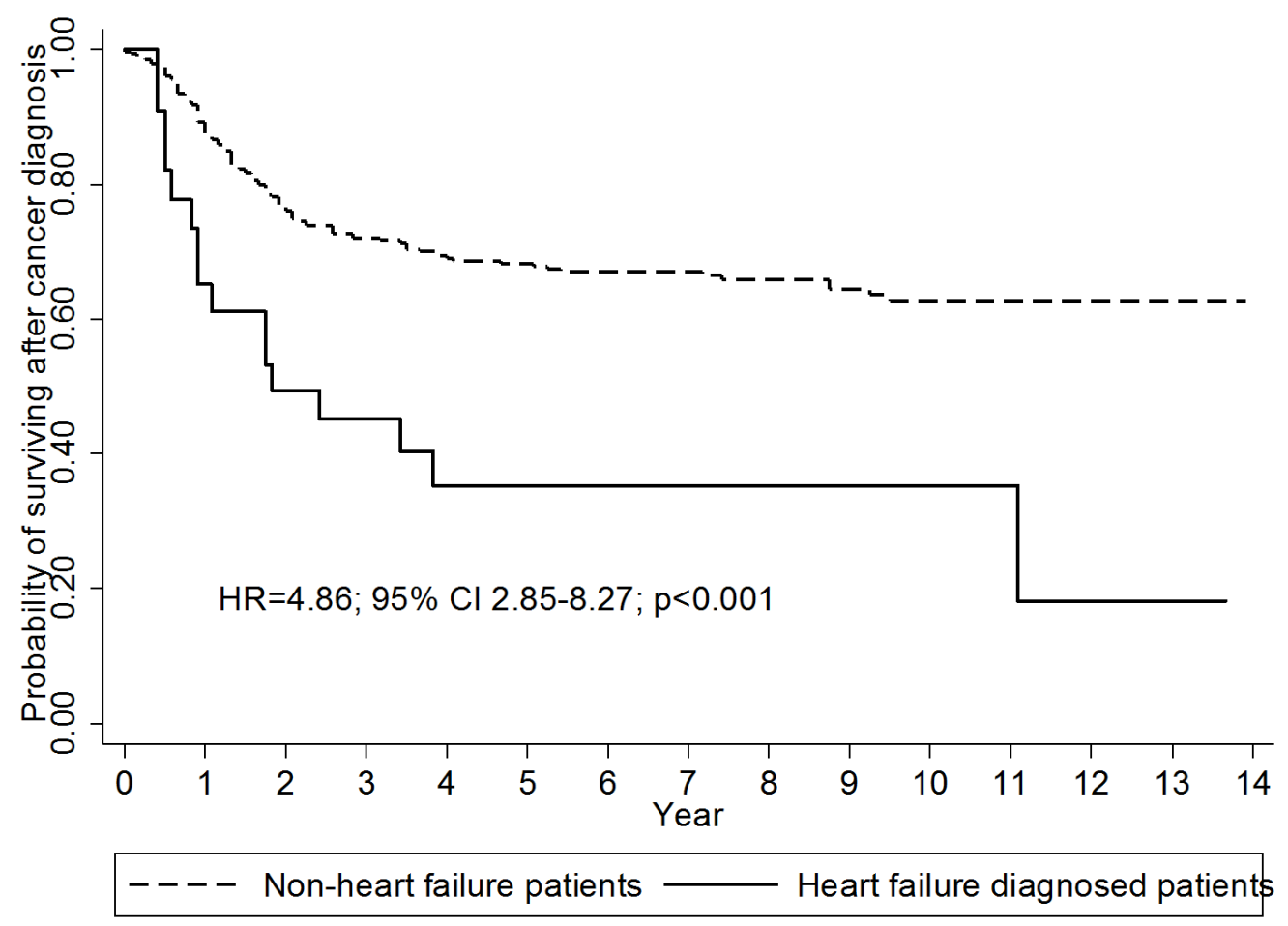

\begin{tabular}{cccccccccccccccc}
\hline $\begin{array}{c}\text { Numbers at } \\
\text { risk }\end{array}$ & 0 & 1 & 2 & 3 & 4 & 5 & 6 & 7 & 8 & 9 & 10 & 11 & 12 & 13 \\
\hline Non-HF & 787 & 693 & 591 & 530 & 472 & 424 & 371 & 317 & 262 & 214 & 163 & 122 & 75 & 38 \\
\hline HF & 30 & 22 & 17 & 13 & 10 & 9 & 8 & 6 & 5 & 3 & 3 & 3 & 2 & 1 \\
\hline
\end{tabular}


Figure 3 Survival curves for all-cause mortality of HF and non-HF chemotherapy-receiving adult cancer patients, adjusted by age, sex, marital status, country of birth and number of chemotherapy separations

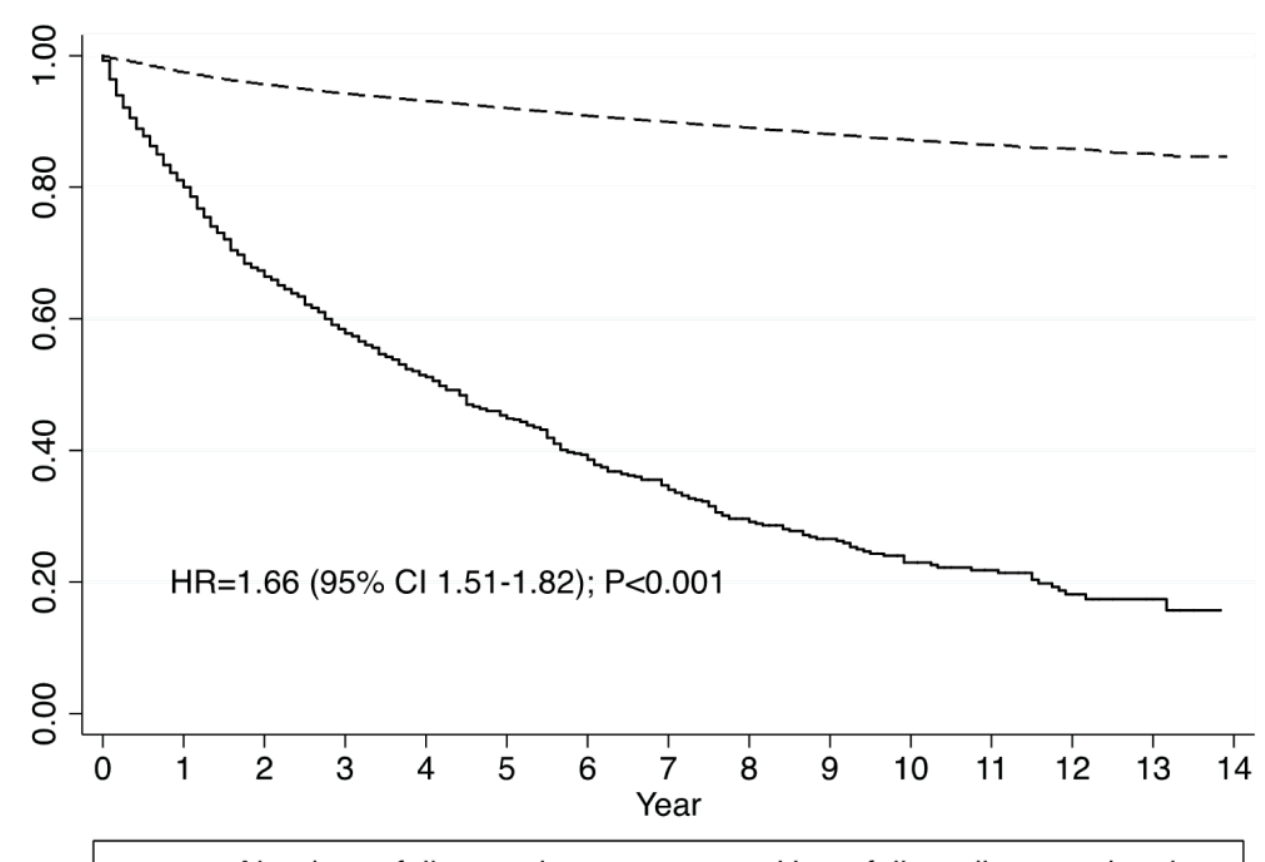

---- Non-heart failure patients $\longrightarrow$ Heart failure diagnosed patients

\begin{tabular}{|c|c|c|c|c|c|c|c|c|c|c|c|c|c|c|}
\hline \multirow[b]{2}{*}{$\begin{array}{c}\text { Numbers at } \\
\text { risk }\end{array}$} & \multicolumn{14}{|c|}{ Year } \\
\hline & 0 & 1 & 2 & 3 & 4 & 5 & 6 & 7 & 8 & 9 & 10 & 11 & 12 & 13 \\
\hline Non-HF & 6,670 & 5,169 & 4,050 & 3,265 & 2,469 & 2,255 & 1,853 & 1,487 & 1,211 & 949 & 716 & 533 & 318 & 153 \\
\hline $\mathrm{HF}$ & 852 & 624 & 468 & 366 & 297 & 242 & 189 & 143 & 112 & 83 & 64 & 45 & 23 & 10 \\
\hline
\end{tabular}


Figure S1 Population selection flow diagram

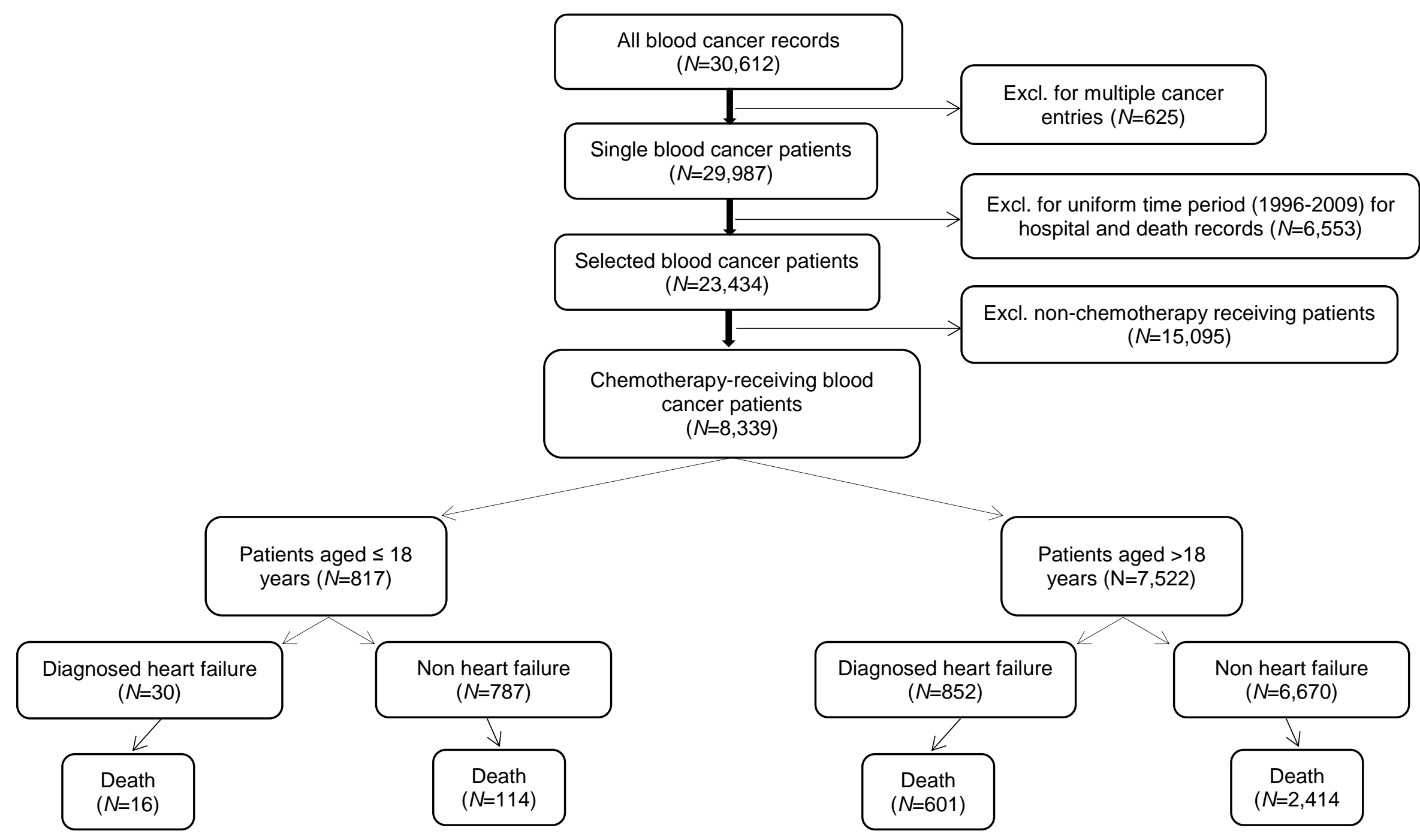

